\title{
Synthesis and Gas Separation Properties of Intrinsic Microporosity Polymer PIM-1
}

\author{
YunHua $\mathrm{Lu}^{1,2, \mathrm{a}^{*}}$, Wei Wang ${ }^{1, \mathrm{~b}}$, Xin $\mathrm{Jin}^{2, \mathrm{c}}$, GuoYong Xiao ${ }^{1, \mathrm{~d}}$, ZhiZhi Hu, \\ TongHua Wang ${ }^{2, f^{*}}$ \\ ${ }^{1}$ School of Chemical Engineering, University of Science and Technology Liaoning, Anshan, \\ Liaoning, China, \\ ${ }^{2}$ School of Chemical Engineering, Dalian University of Technology, Dalian, Liaoning, China \\ alee.lyh@163.com, b949810644@qq.com, `Jinxin925@126.com, `Xiao_guoyong@163.com, \\ ehuzhizhi@163.com, ${ }^{\mathrm{f}}$ wangth@dlut.edu.cn
}

KEY WORDS: PIMs; Polymer membrane; Gas separation; Microporous organic materials Abstract. Polymers of intrinsic microporosity (PIMs) possess rigid and highly non-linear molecular structures. The contortion site of PIM-1 is provided by the monomer 5,5',6,6'-tetrahydroxy-3,3,3',3'-tetramethyl-1,1'-spirobisindane (TTSBI). The paper described the effect of synthetic conditions including catalyst and reaction time on the yield and purity of TTSBI. Then, PIM-1 was synthesized by TTSBI and 2,3,5,6-tetrafluorophthalonitrile in DMF at $60^{\circ} \mathrm{C}$ for 96h. The chemical structure of TTSBI and PIM-1 was characterized by ${ }^{1} \mathrm{H}$ NMR and FT-IR. The gas separation properties of PIM-1 treated in different conditions were obtained. The optimized synthetic condition of TTSBI was that hydrochloric acid was chosen as catalyst to react for five hours at $105^{\circ} \mathrm{C}$. PIM-1 had good solubility in chloroform. The PIM-1 membranes had good gas permeability coefficients for $\mathrm{O}_{2}, \mathrm{~N}_{2}, \mathrm{H}_{2}, \mathrm{CH}_{4}$ and $\mathrm{CO}_{2}$. PIM-1 membrane treated by methanol solvent soaked or redissolved in chloroform showed outstanding gas permeability coefficient and a moderate selectivity.

\section{Introduction}

Growing awareness of the affect of human activities on the world we live in, leads to increasing concern about environmental problems impacting our health and well-being. Membrane separations are seen as a potential technology to tackle several of these problems ${ }^{[1]}$. Further progress in this area necessitates not only the design of novel separation processes, but also the development of new materials with better performance under different conditions, as well as fundamental studies of their performance. This is the theme of the present paper. The preparation of new polymers for use as the selective layer in membranes for gas separations continues to be a significant area of research. Target applications include hydrogen recovery, nitrogen generation, and carbon dioxide capture ${ }^{[2,3]}$.

In recent years, various novel polymeric materials with improved performance (permeability, selectivity) for gas and vapour separations have been developed in order to compete with those currently used $^{[4-7]}$. PIM-1 (polymer of intrinsic porosity 1) was presented by Budd et al. in 2004. Because of its contorted, rigid ladder structure allowing for high free volume, it shows remarkable oxygen permeability (one of the highest). Its synthesis is possible by the reaction of 2,3,5,6-tetrafluorophthalonitrile and TTSBI. 
Centre around PIM-1, the first member of the recently invented ${ }^{[8]}$ class of polymers of intrinsic microporosity (PIMs). PIMs had relied on their own rigid rotation and molecular space structure to obtain a special kind of polymer microporous. Many attempts have been made to quantify the transient and equilibrium sorption of condensable species over the entire vapour activity range in PIM-1 particular on comparing the sorption of vapours of selected PIM-1 solvents. The comparison of the transient sorption of strongly and weakly interacting solvents could help to identify the transport mechanism of organic species in the polymer. And then try to get performance testing of the products. Membranes made of PIMs show outstanding permeability and moderate selectivity. Macroscopically, PIM-1 is a glassy polymer decomposing above $300^{\circ} \mathrm{C}$ without reaching a glass transition $^{[9]}$.

Most of the conformation of polymer has considerable flexibility, its conformation to rearrange the molecular structure of the PIMs and rigidity distortion, to make its main chain can't free rotation. Macromolecules cannot effectively occupy the internal parts of the space, rigid part of the sag to form a continuous microporous PIMs can preparation into insoluble network or soluble polymers.

In this work, 5,5',6,6'-tetrahydroxy-3,3,3',3'-tetramethyl-1,1'-spirobisindane (TTSBI) was obtained by optimizing the synthetic condition. Then, PIM-1 with two phthalocyanine units was prepared by the reactions of TTSBI and 2,3,5,6-tetrafluorophthalonitrile, then converted into membranes by the solvent evaporation method from $8 \mathrm{wt} . \%$ polymer solutions in chloroform. The gas separation properties of PIM-1 treated in various conditions were measured.

\section{Experimental}

Materials. The following chemicals were used as received: 1,2-benzenediol, anhydrous $\mathrm{K}_{2} \mathrm{CO}_{3}$, chloroform, acetic acid, methanol, hydrobromic acid were obtained from Sinopharm Chemical Reagent Co. Ltd.. 2,3,5,6-tetrafluorophthalonitrile was obtained from Aladdin reagent co. LTD.. The reagent $N, N$-dimethylformamide (DMF) was obtained from Tianjin Kemiou chemical reagent co. LTD..

Measurements. Fourier transform infrared (FT-IR) spectroscopy was conducted using a WQF-410 made in the second optical instrument factory, Beijing, China. The transmission measurements were done in a spectral range of 500 to $4,000 \mathrm{~cm}^{-1}$ with a resolution of $4 \mathrm{~cm}^{-1}$ and average of 16 scans. Nuclear magnetic resonance $\left({ }^{1} \mathrm{H}\right.$ NMR) spectra were measured on a $500 \mathrm{MHz}$ AVANCE NMR made in Bruker instrument co. LTD., Switzerland, typically in deuterated dimethyl sulfoxide (DMSO). Molecular weight and molecular weight distributions were measured by gel permeation chromatography (GPC) using tetrahydrofuran (THF) as the fluent. Gas permeation properties were measured using a constant volume variable pressure method at $30^{\circ} \mathrm{C}$ and 0.01 $\mathrm{MPa}^{[10]}$.

Synthesis of $5,5^{\prime}, 6,6^{\prime}$-tetrahydroxy-3,3,3',3'-tetramethyl-1,1'-spirobisindane (TTSBI). A mixture of amount of catalyst, 1,2-benzenediol (33.033 g, $0.30 \mathrm{~mol})$ and acetone (26.136 g, 0.45 mol) in $100 \mathrm{~mL}$ acetic acid was stirred at $105^{\circ} \mathrm{C}$ for several hours. The crude product was cooled and filtered from the solution. The powder was washed with acetic acid and recrystallized by ethanol. The obtained white powder was dried in vacuum for $24 \mathrm{~h}$ at $50^{\circ} \mathrm{C}, \mathrm{mp} .>300 \square$. The TTSBI was characterized by ${ }^{1} \mathrm{H}$ NMR. The synthesis procedure of TTSBI was shown in Scheme 1.

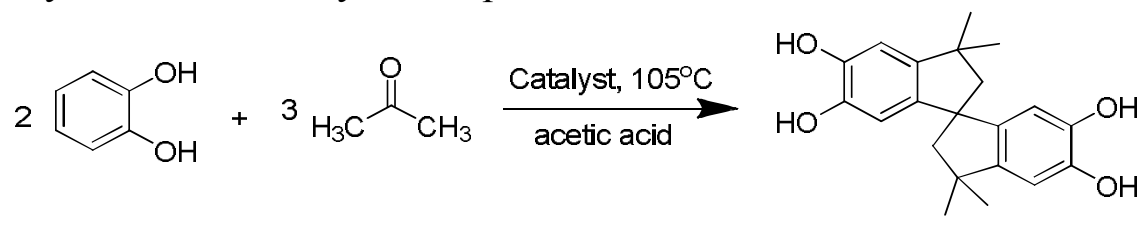

Scheme 1 Synthesis of TTSBI 
Synthesis of PIM-1. A mixture of anhydrous $\mathrm{K}_{2} \mathrm{CO}_{3}$, TTSBI (3.406 $\mathrm{g}, 10 \mathrm{mmol}$ ) and 2,3,5,6-tetrafluorophthalonitrile (TFTPN) $(2.001 \mathrm{~g}, \quad 10 \mathrm{mmol})$ in $35 \mathrm{~mL}$ dry $\mathrm{N}, \mathrm{N}$-dimethylformamide (DMF) was stirred at $60^{\circ} \mathrm{C}$ for $96 \mathrm{~h}$. On cooling, the mixture was added to $100 \mathrm{~mL}$ water, and the crude product was collected by filtration. The crude product was treated in the boiling water for three hours and then filtrated. The product was dried at $50^{\circ} \mathrm{C}$ for $24 \mathrm{~h}$. The product was dissolved in chloroform, and then was precipitated into methanol, giving $2.252 \mathrm{~g}$ (41.7 \% Yield) fluorescent yellow powder. The results of GPC: $\mathrm{Mw}=233,582 \mathrm{Da} ; \mathrm{Mn}=132,974$ $\mathrm{Da} ; \mathrm{D}=\mathrm{Mw} / \mathrm{Mn}=1.757$. The synthesis procedure of PIM-1 was shown in Scheme 2. PIM-1 membrane samples were prepared by solution casting from $8 \mathrm{wt} \%$ polymer solutions in chloroform and dried in covered Teflon circular dishes under ambient temperature and pressure for at least $24 \mathrm{~h}$.

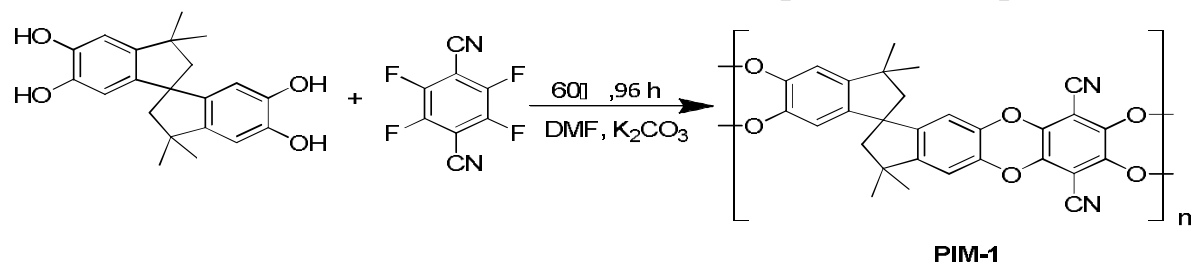

Scheme 2 Preparation of PIM-1 by chemical

\section{Results and discussion}

Optimized synthesis conditions of TTSBI. 1,2-benzenediol (16.517 g, $150 \mathrm{mmol})$ and acetone $(13.068 \mathrm{~g}, 225 \mathrm{mmol})$ were dissolved in $50 \mathrm{~mL}$ acetic acid and three kinds of catalyst $(7 \mathrm{~mL})$ was added and stirred at $105^{\circ} \mathrm{C}$ for $5 \mathrm{~h}$ respectively. The effect of catalyst type on the yield and purity of TTSBI was given in Table 1. When hydroiodic acid (HI) was chosen as catalyst, $4.729 \mathrm{~g} \mathrm{(18.52 \% )}$ TTSBI was obtained with $95.925 \%$ purity (HPLC). When hydrobromic acid (HBr) was chosen as catalyst, $5.900 \mathrm{~g}(23.11 \%)$ TTSBI was obtained with $97.889 \%$ purity (HPLC). When hydrochloric acid $(\mathrm{HCl})$ was chosen as catalyst, there was no product to be obtained. $\mathrm{HBr}$ showed better catalytic effect, which could get high yield and purity of TTSBI.

Table 1 Optimized catalyst of TTSBI

\begin{tabular}{cccc}
\hline Catalyst & $\mathrm{HCl}$ & $\mathrm{HBr}$ & $\mathrm{HI}$ \\
\hline Yield (\%) & 0 & 23.11 & 18.52 \\
Purity (\%)(HPLC) & 0 & 97.889 & 95.925 \\
\hline
\end{tabular}

A mixture of 1,2-benzenediol $(16.517 \mathrm{~g}, 150 \mathrm{mmol})$ and acetone $(13.068 \mathrm{~g}, 225 \mathrm{mmol})$ in $50 \mathrm{~mL}$ acetic acid was stirred at $105^{\circ} \mathrm{C}$ and $7 \mathrm{~mL} \mathrm{HBr}$ was added as catalyst. The yield and purity of TTSBI in different reaction time were given in Table 2. It can be seen that the yield and purity of crude product TTSBI were both high when the reaction time was five hours.

Table 2 Optimized reaction time of TTSBI

\begin{tabular}{ccccc}
\hline Reaction Time (h) & 3.5 & 4.0 & $\mathbf{5 . 0}$ & 6.5 \\
\hline Yield (\%) & 12.39 & 23.11 & $\mathbf{2 8 . 3 0}$ & 21.28 \\
Purity (\%)(HPLC) & 95.915 & 95.448 & $\mathbf{9 8 . 7 9 5}$ & 97.510 \\
\hline
\end{tabular}

Structure of TTSBI and PIM-1. ${ }^{1} \mathrm{H}$ NMR spectra of the monomer TTSBI was shown in Fig. 1. ${ }^{1} \mathrm{H}$ NMR (500 MHz, DMSO- $\left.d_{6}\right): \delta(\mathrm{ppm}): 8.56(\mathrm{~s}, 2 \mathrm{H}), 8.51(\mathrm{~s}, 2 \mathrm{H}), 6.52(\mathrm{~s}, 2 \mathrm{H}), 6.09(\mathrm{~s}, 2 \mathrm{H}), 2.18$ $(\mathrm{d}, J=13.0 \mathrm{~Hz}, 2 \mathrm{H}), 2.02(\mathrm{~d}, J=12.8 \mathrm{~Hz}, 2 \mathrm{H}), 1.27(\mathrm{~s}, 6 \mathrm{H}), 1.21(\mathrm{~s}, 6 \mathrm{H})$. Fig. 2 showed the FT-IR spectra of PIM-1. Formation of PIM-1 is observed in the expected range around $2980 \mathrm{~cm}^{-1}$. It is hydrocarbon stretching vibration on the benzene ring. The spectrum of the PIM-1 also shows 
clearly that the nitrile group is at $2250 \mathrm{~cm}^{-1}$. Ether bond stretching vibration ranged around 1230 $\mathrm{cm}^{-1}$ and $1120 \mathrm{~cm}^{-1}$.

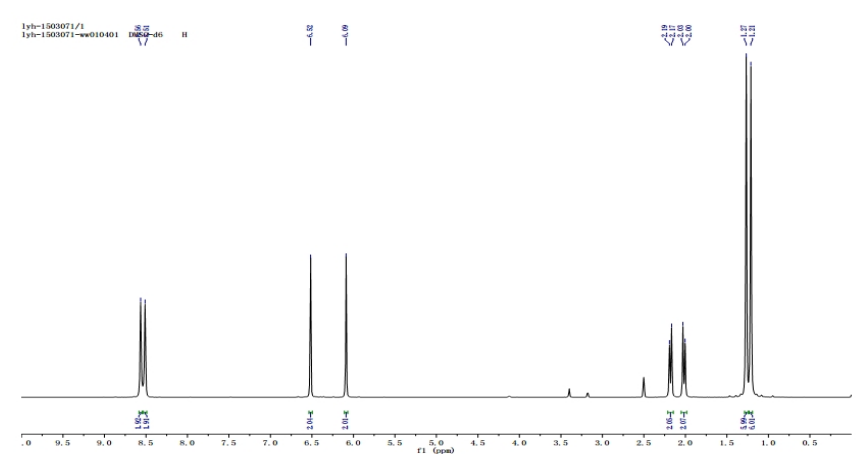

Fig. $1{ }^{1}$ H NMR spectra of TTSBI

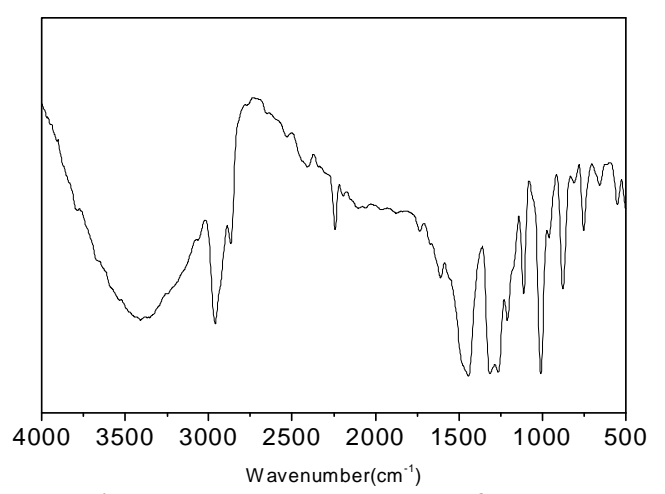

Fig.2 FT-IR spectra of PIM-1

Solubility. The solubility behavior of PIM-1 depended on chain packing density and intermolecular interactions. The same quality of PIM-1 $10 \mathrm{mg}$ was added in $1 \mathrm{~mL}$ of different organic solvent at room temperature for $24 \mathrm{~h}$. PIM-1 had good solubility in dichloromethane and chloroform, especially in chloroform, but PIM-1 didn't dissolve in $N, N$-dimethylformamide (DMF), $N, N$-dimethylacetamide (DMAc), $N, N$-dimethyl sulfoxide (DMSO), methanol, ethanol etc..

Gas separation properties. Gas permeability and selectivity for $\mathrm{O}_{2}, \mathrm{~N}_{2}, \mathrm{H}_{2}, \mathrm{CH}_{4}$ and $\mathrm{CO}_{2}$ were measured for the PIM-1 membranes in different treatment conditions (Table 3). Treatment condition (a) represents that PIM-1 membrane was not further treated by solvent; Treatment condition (b) represents that PIM-1 membrane was soaked in methanol for two hours; Treatment condition (c) represents that PIM-1 membrane was redissolved in chloroform to be membrane.

Table 3 Gas separation properties of the PIM-1 membranes

\begin{tabular}{|c|c|c|c|c|c|c|c|c|c|}
\hline \multirow{2}{*}{$\begin{array}{c}\text { PIM-1 } \\
\text { membranes }\end{array}$} & \multicolumn{5}{|c|}{ Permeability (Barrer $\left.{ }^{a}\right)$} & \multicolumn{4}{|c|}{ Selectivity } \\
\hline & $\mathrm{O}_{2}$ & $\mathrm{~N}_{2}$ & $\mathrm{H}_{2}$ & $\mathrm{CH}_{4}$ & $\mathrm{CO}_{2}$ & $\mathrm{O}_{2} / \mathrm{N}_{2}$ & $\mathrm{CO}_{2} / \mathrm{CH}_{4}$ & $\mathrm{CO}_{2} / \mathrm{N}_{2}$ & $\mathrm{CO}_{2} / \mathrm{H}_{2}$ \\
\hline (a) & 41.91 & 13.96 & 764.25 & 314.42 & 6008.73 & 3.00 & 19.11 & 430.48 & 7.86 \\
\hline (b) & 1939.64 & 815.73 & 5504.13 & 1897.56 & 13055.25 & 2.38 & 6.88 & 16.00 & 2.37 \\
\hline (c) & 1334.47 & 374.47 & 2042.69 & 682.21 & 10096.92 & 3.56 & 14.80 & 29.10 & 4.94 \\
\hline
\end{tabular}

Note: (a) PIM-1 membrane without solvent treatment;

(b) PIM-1 membrane soaked in methanol for two hours;

(c) PIM-1 membrane redissolved in chloroform;

${ }^{\mathrm{a}}$ Barrer $=1 \times 10^{-10} \mathrm{~cm}^{3}(\mathrm{STP}) \mathrm{cm} / \mathrm{cm}^{2} \mathrm{~s} \mathrm{~cm} \mathrm{Hg}^{-1}$

For (a) sample, the permeabilities for $\mathrm{O}_{2}, \mathrm{~N}_{2}, \mathrm{H}_{2}, \mathrm{CH}_{4}$ and $\mathrm{CO}_{2}$ were respectively 41.91, 13.96, 764.25, 314.42 and 6008.73 barrer, while the selectivities for $\mathrm{O}_{2} / \mathrm{N}_{2}, \mathrm{CO}_{2} / \mathrm{CH}_{4}, \mathrm{CO}_{2} / \mathrm{N}_{2}, \mathrm{CO}_{2} / \mathrm{H}_{2}$ were respectively $3.00,19.11,430.48$ and 7.86. After soaking in methanol, the gas permeability coefficient of the (b) sample increased significantly, but the selectivities of the gases were declined. The gas permeability coefficient of (c) sample was also obviously increased. The selectivity for $\mathrm{O}_{2} / \mathrm{N}_{2}$ was 3.56 , but the selectivity for the other gases were declined, especially the selectivity for $\mathrm{CO}_{2} / \mathrm{N}_{2}$ decreased from 430.48 to 29.10 . The reason for it is that (a) sample was dissolved inadequately, leading to low gas permeability coefficients and high selectivities. The gas permeability coefficients of (b) sample were improved significantly because the distance between the molecules was increased by organic solvent swelling. (c) sample showed favourable gas permeability coefficient due to adequate dissolved progress. PIM-1 had very high permeability coefficient for these gases especially for $\mathrm{CO}_{2}$. On the one hand, microporous structure of PIM-1 greatly reduced the resistance when these gas molecules passed through, improved the gas 
permeability coefficient. On the other hand, oxygen atoms of $\mathrm{CO}_{2}$ and polarity heterocyclic atoms of PIM-1 had formed electrostatic interaction each other. The electrostatic interaction made PIM-1's gas permeability coefficient for $\mathrm{CO}_{2}$ significantly increased. The mechanism for $\mathrm{O}_{2}, \mathrm{~N}_{2}, \mathrm{H}_{2}$ and $\mathrm{CH}_{4}$ was mainly molecular sieving. The mechanism for $\mathrm{CO}_{2}$ was mainly solubility-diffusivity and molecular sieving. Therefore, PIM-1 membrane treated by methanol solvent soaked or redissolved in chloroform showed outstanding gas permeability coefficient and a moderate selectivity.

\section{Conclusions}

The optimized conditions for synthesizing TTSBI were given. 1,2-benzenediol and acetone were mixed in acetic acid and hydrochloric acid was added as catalyst at $105^{\circ} \mathrm{C}$ for five hours. The HPLC purity was $98.286 \%$. The structure of TTSBI was characterized ${ }^{1} \mathrm{H}$ NMR. For PIM-1, the formation of PIM-1 was observed by FT-IR. PIM-1 had good solubility in dichloromethane and chloroform. The solvent post-treatment had influences on the gas separation properties of PIM-1. PIM-1 membrane treated by methanol solvent soaked or redissolved in chloroform showed outstanding gas permeability coefficient and a moderate selectivity. Especially for $\mathrm{CO}_{2}$, the permeability of PIM-1 can highly reach at 13055.25 barrer. Therefore, these PIM-1 membranes might be useful for gas or vapor separations.

\section{Acknowledgment.}

This work was financially supported by the National Natural Science Foundation of China (Grant No. 21406102, 21176036, 21376037, 21436009) and China Postdoctoral Science Foundation (Grant No.2014M560212).

\section{References}

[1] P. Bernardo, E. Drioli, G. Golemme, Membrane gas separation. A review/state of the art, Chem. Res. 48 (2009) 4638-4663.

[2] N.W. Ockwig, T.M. Nenoff, Membranes for Hydrogen Separation, Chem. Rev. 107 (2007) 4078-4110.

[3] L. Shao, B.T. Low, T.S. Chung, A.R. Greenberg, Polymeric membranes for the hydrogen economy: contemporary approaches and prospects for the future, J. Membr. Sci. 327 (2009) 18-31.

[4] Y. Yampolskii, B.Freeman (Eds.), Membrane Gas Separation Modelling Gas Separation in Porous Membranes, John Wiley \& Sons, Chichester, England, 2010.

[5] Yu. Yampolskii, I. Pinnau, B.D. Freeman (Eds.), Materials Science of Membranes for Gas and Vapor Separation, John Wiley \& Sons, Chichester, England, 2006.

[6] L.M. Robeson, Correlation of separation factor versus permeability for polymeric Membranes, J. Membr. Sci. 62 (1991) 165-185.

[7] L.M. Robeson, The Upper bound revisited, J. Membr. Sci. 320 (2008) 390-400.

[8] N.B. McKeown, P.M. Budd, K. Msayib, B. Ghanem, Microporous Polymer Material, Int. Pat. 2005, WO05012397.

[9] S. Thomas, I. Pinnau, N. Du, M.D. Guiver, Pure- and mixed- gas permeation properties of a microporous spirobisindane-based ladder polymer (PIM-1), J. Membr. Sci. 333 (2009) 125-131.

[10] C.W. Song, T.H. Wang, X.Y. Wang, J.S. Qiu and Y.M. Cao, Preparation and gas separation properties of poly(furfuryl alcohol)-based C/CMS composite membranes, Sep. Purif. Technol. 58 (2008) 412-418. 